\title{
Oocyte Cryopreservation: Retrospective Analysis of 5 Years Experience
}

\author{
Halime GOKTEPE ${ }^{1}$, Esengul TURKYILMAZ ${ }^{2}$, Gulsen DOGAN DURDAG ${ }^{3}$, Murat SONMEZER ${ }^{4}$, \\ Cem ATABEKOGLU ${ }^{4}$, Batuhan OZMEN ${ }^{4}$
}

Konya, Turkey

\begin{abstract}
OBJECTIVE: To analyze demographic and clinical data of patients who resorted to oocyte freezing between January 2014 and December 2018.

STUDY DESIGN: Patients who applied to the Reproductive Endocrinology and Infertility Unit of Ankara University School of Medicine between January 2014 and December 2018 with the request of oocyte freezing were included in this study. The files and computer records of the patients were analyzed retrospectively and sociodemographic, clinical and laboratory data were evaluated.

RESULTS: A total of 46 cycles were recorded in 40 patients over a 5 -year period. The main indications were low ovarian reserve and/or advanced age $(68.3 \%)$ and malignancy diagnosis $(31.7 \%)$. There was a significant difference between elective fertility preservation and oncofertility preservation (Onco-FP) groups in terms of the age $(38.4 \pm 4.7$ vs $28.4 \pm 6.1 ; p=0.001)$. There was a significant difference between two groups in favor of oncofertility group in terms of anti-Mullerian hormone level, basal follicle-stimulating hormone level, antral follicle count, trigger day estradiol (E2) level, number of obtained oocytes, MII oocytes, and frozen oocytes

CONCLUSION: According to our study, the most prominent oocyte cryopreservation indication was advanced age and/or low ovarian reserve. The number of oocytes collected from patients in the Onco-FP group and thus the number of frozen oocytes was significantly higher than in the elective fertility preservation group, due to younger ages and better ovarian reserve in the Onco-FP group. Abdominal administration of the technique is particularly important for virgin patients in our country. Oocyte freezing is a fertility protection method available in a wide range of indications for reproductive-aged women.
\end{abstract}

Keywords: Cryopreservation, Elective fertility preservation, Oocyte, Oncofertility preservation

Gynecol Obstet Reprod Med 2021;27(3):248-253

${ }^{1}$ Department of Gynecology and Obstetrics, Konya Education and Research Hospital, Konya, Turkey

${ }^{2}$ Department of Gynecology and Obstetrics, Ankara City Hospital, Ankara, Turkey

${ }^{3}$ Department of Gynecology and Obstetrics, Baskent University, Faculty of Medicine, Adana, Turkey

${ }^{4}$ Department of Gynecology and Obstetrics, Ankara University, Faculty of Medicine, Ankara, Turkey

Address of Correspondence: Esengul Turkyilmaz Ankara City Hospital Ankara Turkey

turkyilmaz06@yahoo.com

Submitted for Publication 29.07.2019 Revised for Publication: 23.09.2019 Accepted for Publication: 10.10.2019 Online Published: 01.11.2019

ORCID IDs of the authors: ET: 0000-0003-0873-7528 MS: 0000-0001-6101-1414 BO: $0000-0002-4504-669 X$

HG: 0000-0002-5026-3680

GDD: 0000-0002-5064-5267

CA:0000-0003-0264-0709

\begin{tabular}{|c|c|}
\hline $\begin{array}{c}\text { Quick Response Code: } \\
\text { [ }\end{array}$ & Access this article online \\
\cline { 2 - 2 } & $\begin{array}{l}\text { Website: www.gorm.com.tr } \\
\text { e- mail: info@gorm.com.tr }\end{array}$ \\
\cline { 2 - 3 } & DOI:10.21613/GORM.2019.1001 \\
\hline
\end{tabular}

How to cite this article: Goktepe H. Turkyilmaz E. Dogan Durdag G. Sonmezer M. Atabekoglu C. Ozmen B. Oocyte Cryopreservation: Retrospective Analysis of 5 Years Experience Gynecol Obstet Reprod Med $2021 ; 27(3): 248-253$

\section{Introduction}

Due to social reasons, delayed marriage to advanced years and the presence of patients diagnosed with cancer, the desire to preserve fertility is increasing.

Ovarian capacity and fertility decrease with age in women. This has led to a new approach in the world called "social egg freezing". At the same time, with the improvements in cancer treatment, oocyte freezing has gained importance for patients who are diagnosed with cancer at an early age and desire to maintain fertility. Other factors that reduce fertility include having a poor ovarian reserve, a history of early menopause in the family, systemic disease (Systemic lupus erythematosus, rheumatoid arthritis groups) that have to be put on lifelong immunosuppression and having advanced stage endometriosis that requires oophorectomy and disrupts the quality of life $(1,2)$. Fertility protection procedures are prominent for this group of patients.

Gamete, tissue and embryo storage procedures are deter-

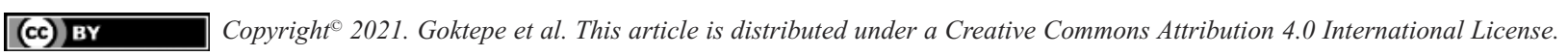


mined by law. Medical laws published in the Official Newspaper on 30 September 2014 in our country, medical necessities are as follows

1. Before treatments that damage gonadal cells such as chemotherapy and radiotherapy,

2. Before surgery (such as removal of the ovaries), which will result in the loss of reproductive functions,

3. Oocyte freezing may be performed in women with low ovarian reserve if there is no child or a family history of early menopause is documented by a medical board report consisting of three specialist physicians (3).

Fertility preservation technologies include embryo freezing, oocyte freezing, and gamete tissue freezing. The choice of fertility preservation method depends on the patient's pubertal status, the presence of the partner, the type and duration of chemotherapy or radiotherapy implemented, and the length of time required for treatment. In addition to the many advantages of embryo freezing, such as storage of surplus embryos, reducing the number of fresh transfers that prevents the risk of multiple pregnancies, and reducing the need for repetitive induction protocols, major disagreements and legal difficulties after the separation between couples might occur because of these embryos (4). Oocyte freezing at an early age may reduce the risk of fetal loss due to age-related aneuploidy. The use of frozen, autologous oocytes also enables the mother to have a genetic relationship with her child, which cannot be obtained through oocyte donation and increases the chance of conception according to the standard in vitro fertilization (IVF) at an older age $(5,6)$. Oocyte freezing may also be an option if enough sperm samples are not obtained on the day of the oocyte pick up. Gamete tissue freezing is currently experimental and has the risks of surgery. However, it can be considered for girls diagnosed with cancer before puberty (7).

While the ASRM (American Society for Reproductive Medicine) reported that oocyte freezing was experimental in 2008, with improvements in cryobiology, they reported that it was a reliable fertility preservation option in 2013 (8). This improvement has led to the introduction of oocyte cryopreservation in the increasing number of IVF clinics worldwide.

The first pregnancy from frozen oocyte came from Chen et al. about 30 years ago, but the success rate did not increase rapidly (9). The reason for this was caused by the method called slow freezing in the oocyte freezing method. The icing occurred in the oocyte in this technique and success rates were low due to the damage of the cryoprotectants used. Thus the studies were conducted over animal experiments for a long time $(10,11)$. With the development of the vitrification technique, by the establishment of a glassy liquid mixture in the oocyte first pregnancy after vitrification came from Kuleshova et al. in 1999. In recent years, with the development of vitrification technique, after freezing and thawing without inducing ice crystals in the oocyte cytoplasm, the damage to the internal organelles of the oocyte has decreased and success rates have been increased. Many IVF programs now support vitrification as a technique of cryopreserving mature oocytes (12-15).

In this study, we aimed to evaluate sociodemographic, clinical and laboratory characteristics of patients undergoing oocyte cryopreservation in our center.

\section{Material and Method}

In this retrospective study, the files of patients who applied to the Unit of Reproductive Endocrinology and Infertility of Department of Obstetrics and Gynecology, Ankara University Faculty of Medicine for oocyte freezing between January 2014 and December 2018 were investigated retrospectively. Patients' files and computer records were examined and age, body mass index, parity, reasons for oocyte freezing, basal hormone profile, anti-Mullerian hormone (AMH) level, antral follicle numbers, previous operation history, a protocol used for ovarian stimulation trigger type used, trigger day estradiol (E2) level, duration and total dose of gonadotropin use, the number of oocytes collected were evaluated. Consent for using data was taken. Ethics committee approval was obtained from Ankara University and numbered with 1-19-19. The study was conducted in accordance with the Declaration of Helsinki.

The files of patients whose data were missing were not processed. Patients who underwent oocyte cryopreservation due to advanced age and/or decreased ovarian reserve were dubbed as elective fertility preservation (EFP), and patients underwent oocyte cryopreservation because of malignancy were dubbed as oncofertility preservation (Onco-FP) group. Since the sociodemographic, laboratory and clinical data were significantly different, the variables were generally examined separately in these two groups and compared with each other. To define decreased ovarian reserve, Bologna criteria were used. In the presence of at least two of the criteria, the patient was diagnosed as "poor ovarian reserve". These criteria are:

1) Advanced maternal age $(\geq 40)$ or any risk factors for poor ovarian reserve

2) Previous poor response history after conventional stimulation ( $\leq 3$ oocytes pick up)

3) Abnormal ovarian reserve tests (Antral follicle count $\leq 5$ or $\mathrm{AMH} \leq 0.5-1.1 \mathrm{ng} / \mathrm{mL}$ )

Mainly, one of the antagonist protocol, progestin priming ovarian stimulation, or antagonist protocol with letrozole/gonadotropin was used. Also, random start stimulation was applied to 2 patients with malignancy. Which protocol will be given to each patient is determined by just the physician's personal preference. Vitrification technique was used to freeze oocytes. 


\section{Antagonist protocol}

Gonadotropin (rFSH and / or HMG) was started on the 2nd or 3rd day of the menstrual cycle. When the leading follicle was $14 \mathrm{~mm}$, the GnRH antagonist was started (Cetrotide 0.25 $\mathrm{mg}$ /day, Merck Serono). Final oocyte maturation was triggered with recombinant human chorionic gonadotropin (rhCG) (Ovitrelle, Merck-Serono, Madrid, Spain) or GnRH agonist $(0.1 \mathrm{mg}$ of Decapeptyl, Ipsen Pharma or $5 \mathrm{mg} / \mathrm{mL}$ Lucrin, Abbott) or a combination of the two as previously described by us when 2 or more follicles are $\geq 18 \mathrm{~mm}$. (16).

\section{Progestin priming ovarian stimulation}

Gonadotropin (FSH and/or HMG) and dydrogesterone (20 $\mathrm{mg}$ /day) were started on the 2 nd or 3rd day of the menstrual cycle until trigger day. Final oocyte maturation was triggered with hCG (rhCG) (Ovitrelle, Merck-Serono, Madrid, Spain) or GnRH agonist (0.1 mg of Decapeptyl, Ipsen Pharma or 5 $\mathrm{mg} / \mathrm{ml}$ Lucrin, Abbott) or a combination of the two when 2 or more follicles are $\geq 18 \mathrm{~mm}$.

\section{Antagonist protocol with letrozole/gonadotropin}

Gonadotropin (FSH and/or HMG) and letrozole (5 $\mathrm{mg}$ /day; Femara; Novartis, Switzerland) were started on the 2 nd or 3 rd day of the menstrual cycle. When the leading follicle was $14 \mathrm{~mm}$, the GnRH antagonists were started (Cetrotide $0.25 \mathrm{mg} /$ day, Merck Serono) per day. Letrozole was continued for 5 days in patients with a low ovarian reserve and continued until trigger day in the group diagnosed with malignancy. Final oocyte maturation was triggered with hCG (rhCG) (Ovitrelle, Merck-Serono, Madrid, Spain) or GnRH agonist $(0.1 \mathrm{mg}$ of Decapeptyl, Ipsen Pharma or 5 $\mathrm{mg} / \mathrm{mL}$ Lucrin, Abbott) or a combination of the two when the leading follicle is about $20 \mathrm{~mm}$.

\section{Statistical Analysis}

Quantitative data were expressed as mean \pm standard deviation and categorical data as a percent. Data of patients were recorded in The Statistical Package for the Social Sciences version 22 (SPSS.22v) and analyzed accordingly. The variables of the 2 groups were compared using independent sample t-test.

\section{Results}

Between January 2014 and December 2018, 127 patients were admitted to our clinic with the request of oocyte freezing. The files of 40 patients were reached. Forty patients un- derwent 46 cycles. Two of these patients were given 2 cycles and 1 of them had 5 cycles.

Twenty-eight (68.3\%) patients underwent elective fertility preservation due to poor ovarian reserve and /or advanced age. Twelve $(31.7 \%)$ of patients were oncofertility preservation patients due to cancer diagnosis before chemotherapy. Three patients had breast cancer, two had ovarian cancer, five had Hodgkin lymphoma, and two had leukemia. Mean age, BMI and parity values of 40 patients are shown in table I.

While 36 (90\%) patients had no previous operation, 1 had unilateral oophorectomy and $3(7.3 \%)$ had endometrioma. Patients with previous endometrioma operations are also those with low ovarian reserve. Six oocytes were collected from one of these patients and 1 oocyte from two of them.

When 46 cycles were evaluated, oocytes could not be collected and frozen in 6 cycles. For 1 of these cycles, gonadotropins were not used for economic reasons, and only the aromatase inhibitor letrozole was used. The number of oocytes collected in 46 cycles was $4.78 \pm 5.9$. The mean number of frozen oocytes was $4.78 \pm 5.9$. The average number of M2 oocytes was $3.69 \pm 4.3$.

The clinical and laboratory values of the Onco-FP and EFP groups in 46 cycles are shown in table II. There was a significant difference between two groups favor of the patients diagnosed with malignity in terms of anti-Mullerian hormone levels, basal FSH level, antral follicle count, trigger day estradiol (E2) value, the total number of obtained oocytes, MII oocytes and, frozen mature oocytes (Table II).

Gonadotropin was not used in 3 cycles of 46 cycles. There was no significant difference between the two groups in terms of total gonadotropin dose, duration of gonadotropin use and duration of antagonist use (Table II).

Antagonist protocol with 63\% (29) cycles is the most commonly used protocol. Antagonist protocol was used in 19 (41\%) cycles in the EFP group and $10(21 \%)$ cycles in the Onco-FP group. Progestin priming ovarian stimulation was used in $7(15.2 \%)$ cycles. Six cycles were the EFP group, 1 cycle was the Onco-FP group. Since one patient had a low ovarian reserve and could not afford gonadotropin, letrozole was preferred for this patient and 4 oocytes were collected in 3 cycles $(6.5 \%)$. Two oocytes were picked up in one cycle and 1 oocyte was picked up in other cycles.

Table I: Sociodemographic variables of patients

\begin{tabular}{lcccc}
\hline Sociodemographic variables & $\begin{array}{c}\text { All patients } \\
(\text { Mean } \pm \text { SD) } \\
\text { Total } n=40\end{array}$ & $\begin{array}{c}\text { Elective FP } \\
(\text { Mean } \pm \text { SD) } \\
\text { Total } n=28\end{array}$ & $\begin{array}{c}\text { Onco-FP } \\
(\text { Mean } \pm \text { SD) }\end{array}$ \\
\hline Age (years) & $35.4 \pm 6.9(40)$ & $38.4 \pm 4.7(28)$ & $28.4 \pm 6.1(12)$ & 0.001 \\
BMI & $22.1 \pm 3.2(34)$ & $21.8 \pm 3(23)$ & $22.6 \pm 3.5(11)$ & 0.5 \\
Parity & 0 & 0 & 0 & NS \\
\hline
\end{tabular}

FP: Fertility preservation, SD: Standard deviation, $n$ : Number of patients, BMI: Body mass index 
Table II: Clinical and laboratory variables of 46 cycles

\begin{tabular}{lccc}
\hline Clinical and laboratory variables (Unit) & $\begin{array}{c}\text { Elective FP (Mean } \pm \text { SD) } \\
\text { Total } n=34\end{array}$ & $\begin{array}{c}\text { Onco-FP (Mean } \pm S D) \\
\text { Total } n=12\end{array}$ & $p$ \\
\hline AMH $(\mathrm{ng} / \mathrm{mL})$ & $0.52 \pm 0.49(\mathrm{n}=29)$ & $1.8 \pm 1.3(\mathrm{n}=9)$ & 0.04 \\
AFC & $3.76 \pm 2.4(34)$ & $8.92 \pm 2.7(12)$ & 0.001 \\
Basal FSH level $(\mathrm{mlU} / \mathrm{mL})$ & $13.7 \pm 6.5(32)$ & $7.3 \pm 3.5(11)$ & 0.002 \\
E2 level on the trigger day $(\mathrm{pg} / \mathrm{mL})$ & $609 \pm 878(29)$ & $11 \pm 8.1(12)$ & 0.005 \\
Number of obtained oocytes & $2.4 \pm 2.1(33)$ & $8.5 \pm 5.5(12)$ & 0.004 \\
Number of obtained MII & $1.91 \pm 1.7(33)$ & $11 \pm 8.1(12)$ & 0.001 \\
Frozen oocyte number & $2.3 \pm 2(32)$ & $2188 \pm 774(12)$ & $\mathrm{NS}$ \\
Total dose of gonadotrophin (IU) & $2301 \pm 1721(29)$ & $8.6 \pm 3.3(12)$ & $\mathrm{NS}$ \\
Duration of gonadotropin use (day) & $8.5 \pm 4.1(33)$ & $4 \pm 2.2(12)$ & $\mathrm{NS}$ \\
Duration of antagonist use (day) & $2.5 \pm 2.5(33)$ &
\end{tabular}

FP: Fertility preservation, SD: Standard deviation, n: Number of patients, AMH: Anti-Mullerian hormone, AFC: Antral follicle count, FSH: Follicle-stimulating hormone, E2: Estradiol, MII: Metaphase II

In 5 cycles $(10.9 \%)$ in the EFP group, despite ovulation induction with gonadotropin follicle growth was not sufficient and the antagonist was not used because of low E2 level. Stop cycle protocol was applied for 2 cycles $(4.4 \%)$ in the EFP group. Initially, since the follicle didn't develop, the protocol was terminated. Ovarian hyperstimulation was restarted if follicle growth was observed one week later. In the Onco-FP group, an aromatase inhibitor (letrozole) was used in addition to gonadotropin. Due to the small number of patients, the results were not compared in terms of protocols used between the EFP and Onco-FP groups.

In 46 cycles, hCG alone was used in 25 patients, GnRH agonist was used in 9 patients, and dual trigger (hCG $+\mathrm{GnRH}$ agonist) was used in 8 patients for oocyte final maturation.

Three patients were not triggered because of no follicle growth. Trigger data of 1 patient couldn't be reached. Although ovarian stimulation was performed in 6 patients in 46 cycles, oocytes could not be collected due to empty follicles or premature ovulation in these cycles.

Oocytes were collected by an abdominal route in 5 cycles (11\%) and by vaginal route in 41 cycles $(89 \%)$.

The pregnancy data couldn't be obtained yet, because the patients who freeze their oocytes didn't return for thawing and in vitro fertilization.

\section{Discussion}

In our study, the majority of the patients were women who requested oocyte cryopreservation due to advanced age and/or low ovarian reserve. Although oocyte freezing is initially of interest in cancer patients, it has been widely used in many medical conditions that may reduce fertility, such as endometriosis, premature ovarian failure, or women who postpone maternity. A new indication under the name of social freezing has begun to be accepted for patients who have a pregnancy plan in older ages and postpone maternity.

In a recent report of the largest series to date, 137 out of 1468 women who underwent elective oocyte cryopreservation for non-oncological reasons returned to use their oocytes. This study showed that pregnancy rates are age-dependent and optimally stored MII oocytes must be at least 8-10 to achieve pregnancy (17). Leading indication for oocyte freezing in our center is low ovarian reserve due to advanced age. However, the mean number of oocytes frozen in these women is $2.3 \pm 2$, which is less than the optimum number of 8 . In such patients, ovarian hyperstimulation multiple times and stimulation protocols involving both follicular and luteal phases may be considered to reach a sufficient number.

In a study of 108 breast cancer patients undergoing fertility preservation between 2005 and 2010, 16.7\% preferred oocyte freezing (18). In another study, oocyte cryopreservation was performed in $71.6 \%$ of cancer patients who requested to preserve fertility. Currently, there are very few reports about the rate of cancer patients returning to use oocytes, but successful live births have been reported (19). In our study, the rate of patients who underwent oocyte cryopreservation due to cancer in all cycles was $31.7 \%$. Similar to previous studies, the low ovarian reserve was not detected in this group of patients $(20,21)$. The number of oocytes collected from patients in the Onco-FP group and thus the number of frozen oocytes was significantly higher than in the EFP group. This is because the mean age of the patients in the cancer group was significantly younger.

According to the number of thawed eggs and freezing methods, live birth rates are different for all ages. A 30-yearold woman with 6 oocytes has a $24.1 \%$ chance of live birth when a 40-year-old woman with 6 oocytes has a $13.4 \%$ chance of live birth after vitrification (22). Female age is the 
main variable determining the success of pregnancy after oocyte resolution and decreases significantly after 35 years of age (23). The contribution of each oocyte dissolved after 41 years to the success of pregnancy decreases thoroughly $(24,25)$. Wennberg et al. defined the cumulative live birth rate as $0 \%$ in those aged $\geq 40$ years (26). In our study, in the elective fertility preservation group, the average age at the time of oocyte cryopreservation was 38.4 years, and the age at the time of egg thawing would be further advanced, thus the success rates would decrease. For this reason, it is important to inform the patient about elective oocyte cryopreservation success rates and limitations considering the possibility of pregnancy in the literature. In addition, some of these women may have maternal-fetal risks in terms of pregnancy due to medical problems they have at the time of oocyte resolution. As recommended by Bachman et al, the detailed informing of these patients must be done at the time of oocyte cryopreservation (27).

In our study, there was no significant difference between the EFP group and the Onco-FP group in terms of the total gonadotropin dose use. EFP could be expected to have a higher total dose of gonadotropin due to containing advanced age patients. However, because of the retrospective nature of the study, the lack of gonadotropin dose in 2 patients, the use of letrozole alone in 3 cycles, and the fact that the dose of gonadotropin treatment did not exceed 225 IU daily in patients with poor ovarian reserve in our clinic may be the reasons for not finding a significant difference between the groups.

In our clinic, the collection of oocytes has also been performed by the abdominal route. Due to lack of data, although the number of all abdominal oocyte pick up (OPU) cases were not clear, abdominal OPU could be performed in $11 \%$ of the patients and obtained oocytes were frozen. In countries where virginity is socially important, such as in our country, the option of abdominal oocyte retrieval is particularly important for patients who are confronted with conditions that require fertility protection, such as cancer diagnosis at an early age.

The limitations of our study are retrospective nature and missing data in the files and computer patient system. Therefore, a limited number of data could be analyzed. In addition, there has not been any demand from our patients for thawing and embryo formation thus far.

Perhaps the pregnancy rates in these patients will be shared in the following years. Cost analysis can only be carried out after years.

Oocyte freezing is a fertility protection method available in a wide range of indications for reproductive-aged women. Cryopreservation of embryos by IVF is a commonly used method of fertility preservation but requires a male partner. Oocyte freezing will be a gateway to the future for women who cannot cryopreserve embryos or who do not want to cry- opreserve embryos, who have fertility-reducing factors, especially cancer patients, patients with a low ovarian reserve and who want to delay childbearing. Oocyte freezing option should be mentioned in risky patient groups and pregnancy rates after oocyte freezing in our country will be shared in the following years.

\section{Acknowledgment: None}

Conflict of interest: The authors reported that there is no conflict of interest.

Funding: There is no funding.

Author Contributions: HG: Data collecting, concept, writing. ET: Data processing, analysis, interpretation, writing. MS: Supervision, concept, design. CA: Supervision, design. BÖ: supervision, analysis.

\section{References}

1. Schattman GL. Clinical practice. Cryopreservation of oocytes. N Engl J Med. 2015;373(18):1755-60. Doi: 10.1056/NEJMcp1307341.

2. Danis RB, Pereira N, Elias RT. Random start ovarian stimulation for oocyte or embryo cryopreservation in women desiring fertility preservation prior to gonadotoxic cancer therapy. Curr Pharm Biotechnol. 2017;18(8):60913. Doi:10.2174/1389201018666170808122531.

3. https://www.resmigazete.gov.tr/eskiler/2014/09/20140930 -4.htm. Say1 29135/sayfa 18.

4. Pennings G. What are the ownership rights for gametes and embryos? Advance directives and the disposition of cryopreserved gametes and embryos. Hum Reprod. 2000; 15(5):979-86. Doi:10.1093/humrep/15.5.979.

5. de Jong A, Dondorp WJ, de Wert GM. The scope of prenatal diagnostic testing for chromosomal aberrations: broad or narrow? Ethical considerations on the choice of tests. Ned Tijdschr Geneeskd. 2009;153:A1060. PMID: 20015413.

6. Goold I, Savulescu J. In favour of freezing eggs for nonmedical reasons. Bioethics. 2009;23(1):47-58. Doi:10. 1111/j.1467-8519.2008.00679.x.

7. Rivas Leonel EC, Lucci CM, Amorim CA. Cryopreservation of human ovarian tissue: a review. Transfus Med Hemother. 2019;46(3):173-81. Doi:10. 1159/000499054.

8. Practice Committees of American Society for Reproductive Medicine, Society for Assisted Reproductive Technology. Mature oocyte cryopreservation: a guideline. Fertil Steril. 2013; 99(1):37-43. Doi:10. 1016/j.fertnstert.2012.09.028.

9. Chen C. Pregnancy after human oocyte cryopreservation. Lancet. 1986;1(8486):884-6. Doi:10.1016/s0140-6736 (86)90989-x.

10. Bernard A, Fuller BJ. Cryopreservation of human oocytes: current problems and perspectives. Hum Reprod. 1996;2 
(3):193-207. Doi:10.1093/humupd/2.3.193.

11. Vincent C, Pickering SJ, Johnson MH. The hardening effect of dimethylsulphoxide on the mouse zona pellucida requires the presence of an oocyte and is associated with a reduction in the number of cortical granules present. $\mathrm{J}$ Reprod Fertil. 1990;89(1):253-9. Doi:10.1530/jrf.0. 0890253.

12. Kuleshova L, Gianaroli L, Magli C, Ferraretti A, Trounson A. Birth following vitrification of a small number of human oocytes: case report. Hum Reprod. 1999;14(12): 3077-9. Doi:10.1093/humrep/14.12.3077.

13. Smith GD, Serafini PC, Fioravanti J, Yadid I, Coslovsky $\mathrm{M}$, Hassun P, et al. Prospective randomized comparison of human oocyte cryopreservation with slow-rate freezing or vitrification. Fertil Steril. 2010;94(6):2088-95. Doi:10. 1016/j.fertnstert.2009.12.065

14. Rudick B, Opper N, Paulson R, Bendikson K, Chung K. The status of oocyte cryopreservation in the United States. Fertil Steril. 2010;94(7):2642-6. Doi: 10.1016/j.fertnstert. 2010.04.079.

15. Chian RC, Huang JY, Tan SL, Lucena E, Saa A, Rojas A, et al. Obstetric and perinatal outcome in 200 infants conceived from vitrified oocytes. Reprod Biomed Online. 2008;16(5):608-10. Doi:10.1016/s1472-6483(10)60471-3.

16. Şükür YE, Özmen B, Özdemir ED, Seval MM, Kalafat E, Sönmezer M, et al. Final oocyte maturation with two different GnRH agonists in antagonist co-treated cycles at risk of ovarian hyperstimulation syndrome. Reprod Biomed Online. 2018; 25(12):1649-54. Doi:10.1016/j. rbmo.2016.10.004.

17. Cobo A, García-Velasco JA, Coello A, Domingo J, Pellicer A, Remohí J. Oocyte vitrification as an efficient option for elective fertility preservation. Fertil Steril. 2016;105(3):755-764.e8. Doi:10.1016/j.fertnstert.2015. 11.027 .

18. Kim J, Oktay K, Gracia C, Lee S, Morse C, Mersereau JE. Which patients pursue fertility preservation treatments? A multicenter analysis of the predictors of fertility preservation in women with breast cancer. Fertil Steril. 2012;97 (3):671-6. Doi:10.1016/j.fertnstert.2011.12.008.

19. Garcia-Velasco JA, Domingo J, Cobo A, Martínez M, Carmona L, Pellicer A. Five years' experience using oocyte vitrification to preserve fertility for medical and nonmedical indications. Fertil Steril. 2013;99(7):1994-9. Doi:10.1016/j.fertnstert.2013.02.004.

20. Cobo A, García-Velasco J, Domingo J, Pellicer A, Remohí J. Elective and Oncofertility preservation: factors related to IVF outcomes. Hum Reprod. 2018;33(12): 2222-31. Doi: 10.1093/humrep/dey321.

21. Dolinko AV, Farland LV, Missmer SA, Srouji SS, Racowsky C, Ginsburg ES. Responses to fertility treatment among patients with cancer: a retrospective cohort study. Fertil Res Pract. 2018;4:3. Doi:10.1186/s40738018-0048-2.

22. Cil AP, Bang H, Oktay K. Age-specific probability of live birth with oocyte cryopreservation: an individual patient data meta-analysis. Fertil Steril. 2013;100(2):492-9.e3. Doi:10.1016/j.fertnstert.2013.04.023.

23. Iussig B, Maggiulli R, Fabozzi G, Bertelle S, Vaiarelli A, Cimadomo D, et al. A brief history of oocyte cryopreservation: arguments and facts. Acta Obstet Gynecol Scand. 2019;98(5):550-8. Doi:10.1111/aogs.13569.

24. Drakopoulos P, Blockeel C, Stoop D, Camus M, de Vos $\mathrm{M}$, Tournaye $\mathrm{H}$, et al. Conventional ovarian stimulation and single embryo transfer for IVF/ICSI. How many oocytes do we need to maximize cumulative live birth rates after utilization of all fresh and frozen embryos? Hum Reprod. 2016;31(2):370-6. Doi:10.1093/humrep/ $\operatorname{dev} 316$.

25. Devesa M, Tur R, Rodriguez I, Coroleu B, Martinez F, Polyzos NP. Cumulative live birth rates and number of oocytes retrieved in women of advanced age. A single centre analysis including 4500 women $>/=38$ years old. Hum Reprod. 2018;33(11):2010-7. Doi:10.1093/humrep/ dey 295.

26. Wennberg AL, Schildauer K, Brännström M. Elective oocyte freezing for nonmedical reasons: a 6-year report on utilization and in vitro fertilization results from a Swedish center. Acta Obstet Gynecol Scand. 2019;98(11):1429-34. Doi:10.1111/aogs.13673.

27. Bachmann G, MacArthur TA, Khanuja K. Need for comprehensive counseling in women requesting oocyte cryopreservation. J Womens Health (Larchmt). 2018;27(3): 227-30. Doi:10.1089/jwh.2017.6423. 